
\title{
28 Research Square \\ Epidemiology and Risk Factors for Resistance to Treatment of Kawasaki Disease in Cyprus.
}

Maria G Koliou ( $\nabla$ koliou-mazeri.maria@ucy.ac.cy )

University of Cyprus Medical School: Panepistemio Kyprou latrike Schole https://orcid.org/0000-00030647-9967

\section{Athina Aristidou}

Archbishop Makarios Hospital, Nicosia

\section{Stella Mazeri}

The University of Edinburgh The Roslin Institute

\section{Elena Georgiou}

Limassol General Hospital: Geniko Nosokomeio Lemesou

\section{Maria Agathocleous}

Limassol General Hospital: Geniko Nosokomeio Lemesou

\section{Marianna Kousparou}

Archbishop Makarios Hospital, Nicosia

\section{Avraam Elia}

Archbishop Makarios Hospital, Nicosia

Antonis Jossif

Pedi Pediatric Cardiology clinic

\section{Research Article}

Keywords: acquired heart disease, Egami score, Kawasaki Disease, Kobayashi score, Resistance to IVIG treatment

Posted Date: December 28th, 2021

DOI: https://doi.org/10.21203/rs.3.rs-1150812/v1

License: @ (i) This work is licensed under a Creative Commons Attribution 4.0 International License. Read Full License 


\section{Abstract}

Background: Kawasaki disease (KD) is one of the most common vasculitides of early childhood and the first cause of acquired heart disease in developed countries. There are no previous studies on KD in Cyprus. The aim of this study was to evaluate the epidemiology of KD in Cyprus, risk factors for resistance to treatment and the development of cardiac complications. Lastly, the Kobayashi and Egami scoring systems were evaluated in our population.

Methods: This is a retrospective multicenter study of pediatric patients with KD hospitalized between January 2000 and-December 2019. The data were collected from medical records.

Results: A total of 136 patients with KD were included in the study. $83 \%$ of patients were $<5$ years of age and $10 \%$ were $<6$ months. Median duration of fever before treatment was 6 days. 108/129 patients responded to initial treatment with IVIG. Thirty patients (22\%) developed coronary artery lesions. Serum sodium $\leq 133 \mathrm{mmol} / \mathrm{L}$, albumin $\leq 3.2 \mathrm{~g} / \mathrm{dl}, \mathrm{ALT} \geq 80 \mathrm{U} / \mathrm{L}$ and neutrophils percentage $\geq 80 \%$ at diagnosis, were identified as risk factors for resistance to IVIG. The Kobayashi and Egami scoring systems had low sensitivity of $57 \%$ and $62 \%$ respectively and a medium specificity of $76 \%$ and $76 \%$ respectively in predicting IVIG resistance.

Conclusion: Clinical and epidemiological characteristics of KD in Cyprus population were similar to those reported in the literature. Although the majority of cases received appropriate treatment in time, cardiac complications still occurred. The Kobayashi and Egami scoring systems cannot be used in our population in order to predict responsiveness to IVIG.

\section{Background}

Kawasaki disease (KD) is one of the most common vasculitides of early childhood and the leading cause of acquired heart disease in children in developed countries [1]. The majority of patients are younger than 5 years old. KD is very rarely encountered in adults. The disease was initially described by Tomisaku Kawasaki, a Japanese pediatrician, in 1967 [2]. Japanese children have been affected more frequently having the highest incidence of the disease which seems to increase over time. Children populations in Korea and Taiwan are following, whereas the incidence in European countries and in the United States of America is much lower [3.4]. In other countries, the disease incidence remains higher in children with Japanese origin in comparison to the rest of the children population which shows that genetic factors may play an important role in the incidence of disease [4]. More than fifty years have passed since the disease was first described, yet the disease etiology remains unknown. There are many theories about the pathogenesis of KD, including an unknown infectious triggering factor [4].

As a result of the unknown etiology of the disease, diagnosis depends on clinical criteria, which include fever, changes of lips and oral cavity, bilateral conjunctival injection, cervical lymphadenopathy, changes in the extremities and rash. The diagnosis becomes doubtful and challenging in atypical- incomplete 
cases where not all of the clinical criteria are met. In such cases the use of laboratory tests can be helpful in supporting the diagnosis [5].

$\mathrm{KD}$ is an acute disease characterized by fever and inflammation of small and medium size arteries. Without treatment, it is a self-limited condition with an average duration of 12 days. However, about 15$25 \%$ of those who remain untreated may develop coronary artery lesions. Up to $50 \%$ of them may develop transient coronary dilation and approximately $25 \%$ may develop more persistent coronary artery abnormalities (CAA's) such as aneurysms [6,7]. Other cardiac complications may be pericardial effusion, myocarditis and valvular regurgitation [5]. Moreover, in patients who receive timely treatment with intravenous immunoglobin (IVIG), only $4 \%$ develop cardiac artery abnormalities.

According to AHA guidelines, the most appropriate treatment is a single dose of IVIG $(2 \mathrm{~g} / \mathrm{kg})$, concurrently with acetylsalicylic acid. A percentage of cases, despite this first-line treatment, are unresponsive. An IVIG non-responsive patient is defined as the patient whose fever persists for more than $36 \mathrm{~h}$ after the end of IVIG infusion [5].

Research on factors associated with non-responsiveness to the administration of initial treatment with IVIG carried out in Japan, showed that non-responsiveness was associated with several parameters such as the young age of patient, the number of days of illness before administration of treatment and laboratory findings recorded before treatment with IVIG, such as high level of C Reactive Protein (CRP), alanine aminotransferase (ALT), aspartate aminotransferase (AST), percentage of neutrophils in full blood count (FBC), low platelet (Plt) count and serum sodium ( $\mathrm{Na}$ ) before treatment. These parameters were included in two different Scoring systems i.e. the Kobayashi (KS) and the Egami score (ES), which were formulated in order to predict the possibility of non-responsiveness in Japanese children [8]. These scoring systems have been evaluated in different populations with varying levels of success [9].

CAA's is the most severe complication of KD. Factors associated with CAA's in some studies, are the young age and the male gender, the delay of treatment beyond 10 days and non-responsiveness to the initial dose of IVIG. Studies reveal also laboratory parameters associated with the development of CAA's such as high level of CRP [10].

The aim of this retrospective study is to evaluate the burden, epidemiology and clinical characteristics of Kawasaki disease in the Cyprus population between 2000 and 2019. Further to this, risk factors for resistance to treatment and the development of cardiac complications were explored. Lastly, the Kobayashi (KS) and Egami (ES) scoring systems were evaluated in our population.

\section{Methods}

\section{Data sources and collection.}

Medical records of patients less than 15 years of age, with complete or incomplete KD, hospitalized in Archbishop Makarios Hospital (AMH) and Limassol General Hospital between January 2000 and 
December 2019 were retrospectively reviewed. As Archbishop Makarios Hospital (our hospital), served as the referral center for Pediatric Cardiology services in all Cyprus, patients from other hospitals in Cyprus, were also referred to our hospital for cardiology assessment. In addition, all severe cases of KD from all Cyprus were referred to $\mathrm{AMH}$ for management as it also acts as the tertiary Pediatric referral center in Cyprus. All cases were categorized into complete and incomplete form, according to the American Heart Association Recommendations 2004 [1]. The first day of fever was defined as the onset of disease. Initial treatment of KD was the administration of high dose IVIG $(2 \mathrm{~g} / \mathrm{kg})$ together with high dose aspirin (80$100 \mathrm{mg} / \mathrm{kg} / \mathrm{d}$ ) and when the patient became afebrile for at least two to three days the dose of aspirin was reduced to $3-5 \mathrm{mg} / \mathrm{kg} /$ day. Because this study includes cases in a time frame of 20 years, treatment regimen in some cases varied. While the total dose remained the same, in the first years of the study the IVIG was administered over 2- 5 days instead of one day. Patients who remained febrile 36 hours after administration of initial IVIG, were defined as IVIG non-responders. Treatment was considered as delayed when the first dose of IVIG was given after the 10th day of illness.

A database was created to include all information regarding cases. This database included demographic, clinical and laboratory as well as outcome data of the patients. Data included age, sex, season of admission, nationality, days of fever before treatment, total days of illness. It contained laboratory values before IVIG treatment (Albumin, Gamma -Glutamyl Transferase ( $\mathrm{YGT}$ ), Hemoglobin ( $\mathrm{Hb}$ ), White Blood Cell count (WBC), Percentage of Neutrophils in FBC, CRP, Erythrocyte Sedimentation Rate (ESR), Na, ALT, AST and the highest number of platelets during the course of illness.

A case of complete KD was defined as the presence of fever longer than 4 days and the presence of 4 out of the 5 clinical features that is changes of lips and oral cavity, bilateral conjunctival injection, cervical lymphadenopathy, changes in the extremities and rash. Based on the AHA guidelines a case of incomplete KD was defined as the child with fever $>=5$ days and two or three of the other clinical criteria or infants with fever for at least 7 days duration without other explanation [5].

Our group of pediatric cardiologists classified the coronary artery abnormalities according to the American Heart Association (AHA) guidelines 2004 and updated in 2017 [5] into 4 categories, depending on coronary artery Z-score which was based on assessment of populations of healthy afebrile children:

- Dilation is defined when Z-score is between 2 and 2,5, or if initially $<2$ and then decrease in Z score during follow-up for at least one year.

- Small aneurysm: $\geq 2,5$ to $<5$

- Medium aneurysm: $\geq 5$ to $<10$, and absolute dimension $<8 \mathrm{~mm}$

- Large or giant aneurysm: $\geq 10$, or absolute dimension $\geq 8 \mathrm{~mm}$

The Kobayashi and Egami risk scoring systems were evaluated in order to test their sensitivity and specificity in predicting non- responders to IVIG in our population of patients. They were further evaluated for predicting high risk cases for development of Coronary artery abnormalities (CAA's). 


\section{Statistical analysis}

Data were analyzed using the R statistical software version 3.5.1 [11]. Package ggplot2 was used for all plots [12]. Differences in clinical findings between complete and incomplete cases of Kawasaki disease were assessed using a chi-squared test. Blood test results between the two groups were also compared. In order to test whether there was a difference, Student T-test and U-Mann-Whitney were applied in parametric and nonparametric distributions respectively. Normality was tested by means of Shapiro-Wilk test. A p-value less 0.05 was considered statistically significant.

Logistic regression modeling was used to investigate factors associated with a) resistance to intravenous immunoglobulin and b) development of coronary artery abnormalities. Univariable analysis of all the factors against each outcome variable using Fisher's exact test was used to select variables to be considered in the multivariable analysis. Variables with a p-value of less than 0.2 were considered for inclusion in each model. All variable combinations were considered using R package MuMIn [13]. Multivariable logistic regression models were fit using Firth's bias reduction method from R package logistf [14]. Model selection was based on Second-order Akaike Information Criterion (AICc). Models with an AICc within 2 units of the lowest AICc were averaged to give the final model.

In order to assess the applicability of the Kobayashi and Egami scores in the Cypriot population their ability to correctly predict a) cases resistant to IVIG and b) cases with coronary artery abnormalities was evaluated. Sensitivity, specificity, positive and negative predictive values were estimated using package epiR[15].

\section{Results}

A total of 136 patients with KD were included in the study. Male to female ratio was 1.25:1 and mean age of patients at diagnosis was 2.97 years (ranged from 0.23 to 14.67 years) and the median age 2.29 years. Eighty-three per cent of the patients were under 5 years of age. Only 32 patients $(23 \%)$ were less than 12 months of age, while 14 children (10\%) were younger than 6 months (Fig. 1).

The majority of the cases were diagnosed during winter and spring months (65\%) ( $p=0.0006)$ (Fig. 2). The patients were mainly Greek Cypriots (88\%).

Overall, 88 cases were classified as complete (69\%) and 40 (31\%) as incomplete Kawasaki disease. The duration of fever before administration of IVIG treatment was on average 7.2 days with a median of 6 . In 17 cases diagnosis was delayed and treatment was started 10 days or more after onset of symptoms, with the longest interval between symptom onset and treatment being 22 days. A small percentage $(11 \%)$ of children with KD were started on treatment less than five days after onset of the disease.

\section{Clinical characteristics}


All patients presented with persistent fever for at least 5 days. Overall, changes of lips and/or oral cavity (erythema and cracking of lips, strawberry tongue, erythema of oral and pharyngeal mucosa) were the most common manifestations, seen in $113 / 127$ patients (89\%), followed by skin rash in 109/131 cases (83\%). This included maculopapular, morbilliform, erythematous or urticarial rash. Bilateral bulbar conjunctival injection without exudates was seen in $103 / 128$ patients $(80 \%)$. Changes of extremities were detected in $89 / 126$ cases $(71 \%)$ in this study. Specifically, in early stages of the disease palmar or/and soles edema or/and erythema were noticed, while desquamation of digits developed in the second week of illness. Cervical lymphadenopathy was the least common clinical finding (71/124 cases $-57 \%)$.

Other clinical findings which do not belong to the main criteria for diagnosis were also observed, particularly gastrointestinal (GI), musculoskeletal and central nervous system symptoms. A total of 60 out of 109 patients (55\%) complained about GI symptoms. Diarrhea/abdominal pain/vomiting were the most common features in these patients. Five patients had hepatomegaly, one of them with jaundice (high level of direct bilirubin, acholic stools), and one with ascites. Musculoskeletal symptoms were present in 20/107 (19\%). Arthralgia and less commonly arthritis occurred in 10 and 7 patients respectively, in various large joints. Two of these had difficulty standing up. In addition, central nervous system features developed in $15 / 113$ patients (13.3\%), extreme irritability being the most frequent symptom, followed by lethargy. Three cases, in which lumbar puncture was done, were diagnosed with aseptic meningitis. Unilateral facial nerve paresis was detected in one patient.

In Table 1 the clinical manifestations in complete vs incomplete cases of KD are compared. There were statistically significant differences in all principal criteria of KD. Changes of lips and/or oral cavity remained the most frequent clinical finding in both complete and incomplete cases.

Table 1

Clinical findings in patients with complete and incomplete KD (* Chi squared test).

\begin{tabular}{|llll|}
\hline Classical criteria & Complete KD & Incomplete KD & P value* $^{*}$ \\
\hline Changes of lips/oral & $83(95.4 \%)$ & $27(77.1 \%)$ & 0.002 \\
\hline Bilateral conjunctival injection & $78(90.7 \%)$ & $21(56.8 \%)$ & $<0.001$ \\
\hline Skin rash & $82(93.2 \%)$ & $23(62.2 \%)$ & $<0.001$ \\
\hline Changes of extremities & $76(86.4 \%)$ & $12(34.3 \%)$ & $<0.001$ \\
\hline Cervical lymphadenopathy & $55(66.3 \%)$ & $13(34.2 \%)$ & 0.001 \\
\hline Other features & & & \\
\hline CNS symptoms & $10(13.2 \%)$ & $5(14.7 \%)$ & 0.771 \\
\hline Gl symptoms & $45(60.8 \%)$ & $15(44.1)$ & 0.145 \\
\hline Musculoskeletal & $17(22.7 \%)$ & $3(9.7 \%)$ & 0.264 \\
\hline
\end{tabular}




\section{Laboratory findings}

Regarding the laboratory findings, children with complete KD had significantly lower levels of platelets before treatment in comparison to incomplete KD patients (median value 383 vs $453, p=0.04$ ) and also higher hemoglobin levels (median value $10.5 \mathrm{~g} / \mathrm{l}$ vs $9.8 \mathrm{~g} / \mathrm{l}, \mathrm{p}=0.038$ ). In complete KD patients, liver enzymes values were higher in comparison to incomplete KD (median ALT 62 vs 22, $p=0.001$, median AST 40 vs $29(p=0.026)$ (Table 2$)$.

Table 2

Laboratory findings in Complete and Incomplete KD (+ U-Mann-Whitney, ++ Student T-test).

\begin{tabular}{|c|c|c|c|}
\hline Laboratory parameter & $\begin{array}{l}\text { Complete KD } \\
\text { (Variable Median [IQR] n) }\end{array}$ & $\begin{array}{l}\text { Incomplete KD } \\
\text { (Variable Median [IQR] n) }\end{array}$ & p-value \\
\hline Albumin $\mathrm{g} / \mathrm{dL}$ & $3.5[1.7-6.8] n=68$ & $3.4[2.5-4.3] n=29$ & $0.844^{+}$ \\
\hline ALT U/L & $62[8-720] n=85$ & $22[6-294] n=40$ & $0.001^{+}$ \\
\hline AST U/L & $40[16-946] n=85$ & $29.5[14-375] n=40$ & $0.026^{+}$ \\
\hline CRP mg/dL & $15[0-40] n=81$ & $11.5[1-40] n=38$ & $0.101^{+}$ \\
\hline $\mathrm{ESR} \mathrm{mm} / \mathrm{h}$ & $70.5[6-140] n=86$ & $77.5[16-152] n=40$ & $0.221^{++}$ \\
\hline $\mathrm{Hb}$ g/dl & $10.5[8-12.6] n=81$ & $9.8[5.7-12.7] \mathrm{n}=35$ & $0.038^{++}$ \\
\hline Highest Plts $\times 10^{\wedge} 9 / \mathrm{L}$ & $707.5[392-1375] n=82$ & $738.5[320-1368] n=38$ & $0.526^{+}$ \\
\hline Plts before IVIG & $383[53-1042] n=88$ & $453[21-1368] n=40$ & $0.040^{+}$ \\
\hline $\mathrm{Wbc} \times 10^{\wedge} 9 / \mathrm{L}$ & $15.6[1.5-40.1] n=87$ & $15.9[7.3-37.8] n=40$ & $0.734^{+}$ \\
\hline Numbers and letters $i$ & g to statis & significant values. & \\
\hline
\end{tabular}

A total of $56(41.1 \%)$ patients developed some form of cardiological complication in the acute phase. As shown in Table 3, coronary artery abnormalities were the most frequent cardiological complication $(22.1 \%)$. The majority of them (23/30 patients) developed transient dilations. In one of these cases the patient developed concurrently myocarditis with ventricular dysfunction. Small aneurysms were detected in four cases and medium diameter aneurysms in two patients. In one case, an initial echo revealed a small aneurysm, and on subsequent echocardiographic investigation it further expanded, becoming a giant aneurysm. Pericardial effusion was the second more common complication (18\%). 
Table 3

Cardiological findings in patients with KD.

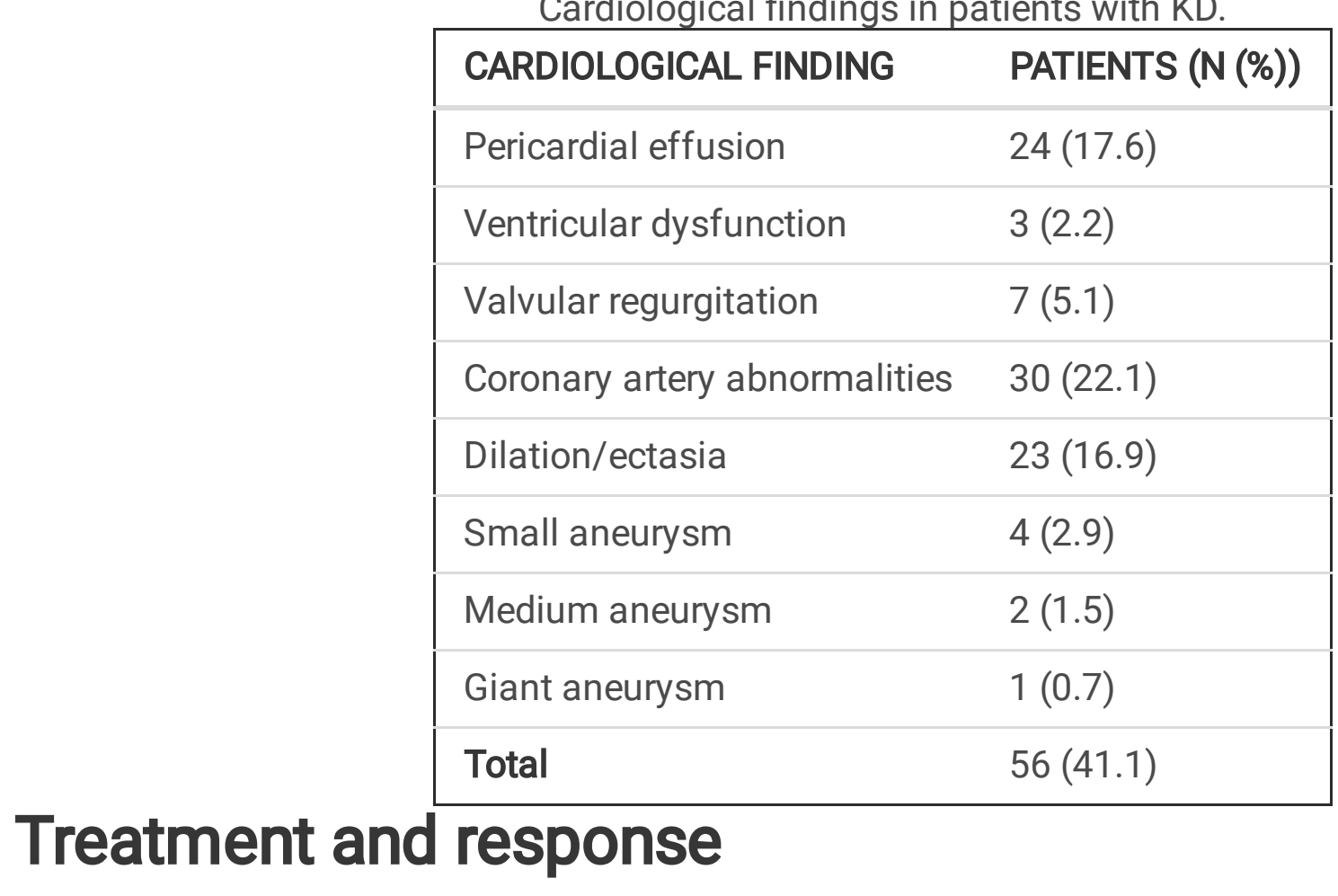

A large proportion of all the patients $(108 / 129-84 \%)$ responded well to the initial treatment with IVIG. Fourteen out of the 21 non-responders became afebrile after the administration of a second dose of IVIG. Five of the remaining seven patients who did not respond to two doses of IVIG responded to a third dose, while two patients required intravenous pulse of methyl prednisolone. One of these two patients additionally required treatment with infliximab, cyclosporin and tocilizumab. The median interval between onset of fever and administration of IVIG was 6 days in complete cases and 6.5 days in incomplete cases (p-value=0.339).

\section{Risk factors for resistance to intravenous immunoglobulin}

A total of 21 patients (16\%) were non-responders to the initial administration of IVIG. A univariable analysis (Additional file 1) was carried out in order to identify clinical and laboratory factors for resistance to IVIG. Non- responders had more commonly lower level of serum albumin and sodium, and higher level of ALT. In our population the age of patients and the day of administration of IVIG were not found to be risk factors for non-responsiveness. Multivariable regression analysis (Fig. 3) suggested that four variables were risk factors for IVIG resistance: Sodium $\leq 133 \mathrm{mmol} / \mathrm{L}$, Albumin $\leq 3.2 \mathrm{~g} / \mathrm{dl}, \mathrm{ALT} \geq 80$ $\mathrm{U} / \mathrm{L}$ and percentage of neutrophils $\geq 80 \%$.

\section{KD and coronary abnormalities}

Overall, 30 patients $(22 \%)$ developed coronary artery abnormalities. Univariable logistic regression analysis (Table 4) suggested that infants, had higher odds of developing CAAs, while children between 1 year and 5 years had statistically significantly lower odds of developing CAAs in comparison to the youngest age group. No significant associations between gender, delayed treatment, the total duration of 
fever and blood parameters such as $\mathrm{Hb}, \mathrm{Na}$, Plts and CAAs were observed in our study population (Additional file 2).

Table 4

Univariable logistic regression of the association between age and CAAs.

\begin{tabular}{|llll|}
\hline Variable & Odds Ratio & $95 \%$ Confidence Interval & P-value \\
\hline Age & & & \\
\hline$<6$ months & 1.0 & Reference value & \\
\hline $6-12$ months & 0.34 & $0.08-1.37$ & 0.129 \\
\hline $12-59$ months & 0.10 & $0.03-0.33$ & $<0.001$ \\
\hline 60 months or older & 0.33 & $0.08-1.31$ & 0.116 \\
\hline
\end{tabular}

Evaluation of the Kobayashi and Egami scoring systems.

38 out of 128 patients with complete data relevant to IVIG resistance and Kobayashi scoring, were identified to have high risk to be non-responders based on the KS (positive when score $\geq 4$ ). Out of those, only 12 patients had IVIG resistance (Table 5). It was found that KS had low sensitivity (57\%) and medium specificity (76\%) in predicting IVIG resistance. Similarly, 37 out of 123 patients were classified as high risk of being non-responders based on ES (positive when score $\geq 3$ ), but only 13 of those actually had IVIG resistance (Table 5). ES was also found to have low sensitivity of $62 \%$ and medium specificity of $76 \%$ to identify non-responders in our population.

Table 5

Cross tabulation of IVIG resistance vs KS and ES results.

\begin{tabular}{|llllllll|}
\hline \multicolumn{2}{|c|}{ Kobayashi score } & \multicolumn{5}{c|}{ Egami score } \\
\hline $\begin{array}{l}\text { IVIG } \\
\text { resistant }\end{array}$ & $\begin{array}{l}\text { Not IVIG- } \\
\text { resistant }\end{array}$ & Total & & $\begin{array}{l}\text { IVIG } \\
\text { resistant }\end{array}$ & $\begin{array}{l}\text { Not IVIG- } \\
\text { resistant }\end{array}$ & Total \\
$\begin{array}{l}\text { KS } \\
+\mathrm{ve}\end{array}$ & 12 & 26 & 38 & $\begin{array}{l}\text { ES } \\
\text { tve }\end{array}$ & 13 & 24 & 37 \\
$\begin{array}{l}\mathrm{KS}- \\
\text { ve }\end{array}$ & 9 & 81 & 90 & $\begin{array}{l}\text { ES }- \\
\text { ve }\end{array}$ & 8 & 78 & 86 \\
\hline Total & 21 & 107 & 128 & Total & 21 & 102 & 123 \\
\hline
\end{tabular}


Table 6

Kobayashi and Egami score in predicting IVIG non-response.

\begin{tabular}{|lll|}
\hline & \multicolumn{3}{|c|}{ Kobayashi Score (95\%Cl) } & Egami Score (95\% Cl) \\
\hline Sensitivity & $57.1 \%(34.0-78.2)$ & $61.9 \%(38.4-81.9)$ \\
\hline Specificity & $75.7 \%(66.5-83.5)$ & $76.5 \%(67.0-84.3)$ \\
\hline PPV & $31.6 \%(17.5-48.7)$ & $35.1 \%(20.2-52.5)$ \\
\hline NPV & $90.0 \%(81.9-95.3)$ & $90.7 \%(82.5-95.9)$ \\
\hline
\end{tabular}

Table 7 shows the cross tabulation of patients with CAA and results of KS and ES using the same cutoffs as mentioned above. KS and ES were found to have poor sensitivity in predicting those patients who developed CAAs of $33.3 \%$ and $29 \%$ respectively (Table 8 ).

Table 7

Cross tabulation of CCA vs KS and ES results.

\begin{tabular}{|llllllll|}
\hline \multicolumn{5}{|c|}{ Kobayashi score } & \multicolumn{5}{c|}{ Egami score } \\
\hline & CAA & Not CAA & Total & & CAA & Not CAA & Total \\
KS +ve & 10 & 27 & 37 & ES +ve & 8 & 28 & 36 \\
KS -ve & 20 & 73 & 93 & ES -ve & 20 & 63 & 83 \\
Total & 30 & 100 & 130 & Total & 28 & 91 & 119 \\
\hline
\end{tabular}

Table 8

Kobayashi and Egami score in predicting development of CAA.

\begin{tabular}{|lll|}
\hline & \multicolumn{3}{|c|}{ Kobayashi Score (95\% Cl) } & Egami Score (95\% Cl) \\
\hline Sensitivity & $33.3 \%(17.3-52.8)$ & $28.6 \%(13.2-48.7)$ \\
\hline Specificity & $73.0 \%(63.2-81.4)$ & $69.2 \%(58.7-78.5)$ \\
\hline PPV & $27.0 \%(13.8-44.1)$ & $22.2 \%(10.1-39.2)$ \\
\hline NPV & $78.5 \%(68.8-86.3)$ & $75.9 \%(65.3-84.6)$ \\
\hline
\end{tabular}

\section{Discussion}

This is the first study on KD carried out in Cyprus using National multicenter data. The epidemiology of the disease in our country, has similar characteristics with the previous studies in other countries especially those sharing the same climatic conditions such as Spain and Greece $[9,16]$. There is a slight preponderance of males (male/female ratio 1.25:1). Children younger than 5 years old are the most frequently affected ( $83 \%$ of cases) with median age of cases 2.23 years, similar to other European countries and different than Japan where the incidence rate was the highest among children aged 6-11 months $[17,18]$. The onset of KD was more frequent in winter and spring $(65 \%)$, which is consistent with 
findings in European reports and in neighboring countries [9]. However, in reports from some Asian countries, the highest incidence rates were reported during summer and spring [19].

The majority of children with KD in Cyprus presented with changes of the lips/oral cavity (89\%), which is in accordance with reports in other countries $[9,16]$. Followed by the skin rash, which considerably varied in nature. More precisely, maculopapular was the most common form, while in two cases a petechial rash was present. Petechial rash is not described as a frequent finding in other studies. Whilst bilateral bulbar conjunctival injection without exudates was the first or second most frequently seen manifestation in some studies [9] in our study it was the third most frequent manifestation noted (80.4\%). Unilateral cervical lymphadenopathy was the least common finding (57\%), which was in accordance with other studies [5].

Diagnosis of complete and incomplete KD depends on clinical and/or laboratory criteria. The rate for incomplete cases in our population was $31 \%$, which is consistent with the rate found in a study in the Spanish population but differs from the rate in a study in Japan where the rate of incomplete disease was only $10 \%[9,20]$. In general, the rate of incomplete $\mathrm{KD}$ is underestimated and is higher in infants than in older age groups $[20,21]$. As expected, all clinical criteria were detected at a significantly lower rate in incomplete vs complete cases. However, similar to complete cases, changes of lips and oral cavity was the most frequent clinical finding in incomplete cases. This was similar to the findings in the Spanish population [9]. Hemoglobin levels on admission were found to be significantly lower in incomplete vs complete cases and AST and ALT were found to be significantly higher. Platelets before administration of IVIG were found higher in incomplete vs complete cases.

The rate of responsiveness to the first dose of IVIG administered is high (84\%) similar to other countries $[5,16,22,23]$. Multivariable regression analysis indicated that four laboratory parameters on admission i.e. sodium $\leq 133 \mathrm{mmol} / \mathrm{L}$, albumin $\leq 3.2 \mathrm{~g} / \mathrm{dl}, A L T \geq 80 \mathrm{U} / \mathrm{L}$ and the percentage of neutrophils $\geq 80 \%$ were positively associated with non-responsiveness to IVIG. There is great variation in risk factors for IVIG nonresponsiveness identified in different studies. A large study in the Spanish population also revealed hypoalbuminemia and hyponatremia as risk factors for non-responsiveness. In addition to these it also revealed anemia and high procalcitonin values as risk factors [9]. In a study in San Diego USA, multivariable analysis revealed early administration of IVIG (before day 5), higher concentrations of YGT, higher \% bands and lower hemoglobin, as risk factors associated with resistance to IVIG [24].

In Japanese children, Kobayashi, the Egami and the Sano scoring systems, have been shown to successfully predict the risk of IVIG resistance $[22,25,26]$. The KS includes some of the risk factors identified in our study such as the sodium $\leq 133 \mathrm{mmol} / \mathrm{L}$, neutrophils $\geq 80 \%$ in the white blood cell count, but also uses some other risk factors such as $A S T \geq 100 \mathrm{U} / \mathrm{L}$, low platelet count $\leq 300 \times 10^{\wedge} 9 / \mathrm{L}$, early administration of IVIG before day 5 of illness and high CRP. Egami scoring system gives scores for age $<6$ months, days of illness $<4$ when administering IVIG, low platelet count, increased CRP and raised ALT. More precisely, the KS has been shown to predict IVIG non-responsiveness with $78 \%$ sensitivity and $76 \%$ specificity, while the Egami score showed $78 \%$ sensitivity and $76 \%$ specificity in Japanese children. In our 
population both scoring systems showed low sensitivity and medium specificity with low positive predictive value (PPV). This was comparable with other non-Japanese populations $[9,27,28]$.

Risk factors for the development of CAAs were evaluated in our study and only young age less than 12 months was identified as a risk factor. Young age $<12$ months was also identified in other studies [29]. However, some other studies have also identified other factors as risk factors for the development of CAAs such as gender, delayed treatment, the total duration of fever and blood parameters [30]. The Kobayashi and Egami scoring systems also showed poor sensitivity in predicting cases with CAAs in our population as was the case in other studies $[9,31]$.

Our study has several strengths and limitations. The retrospective nature of study means it was not possible to collect additional data relevant to Kawasaki disease and that some recorded data may have been missed. However, the records kept were very detailed, in nearly all cases, and contained most data required for the study. The study covers a 19 Year period, providing one of the longest Kawasaki datasets described. The study includes children with KD who have been diagnosed since 2000, therefore the treatment regimen maybe variable in some cases, according to the existing guidelines at the time. However, the total amount of immunoglobulin administered was the same in all cases.

\section{Conclusions}

This retrospective study was the first report on KD in Cyprus. Overall, the epidemiology of the disease, seems to be in accordance with other studies in Europe. Blood parameters such as low level of serum albumin, sodium, high level of ALT and high percentage of neutrophils in the FBC, were found as risk factors for non-responsiveness. Age was the only risk factor identified for the development of CAAs in our study. KS and ES had poor accuracy in predicting IVIG non-responders as well as the patients who developed CAA's.

\section{List Of Abbreviations}

KD, Kawasaki disease; KS, Kobayashi scoring system; ES, Egami scoring system; CAAs, Coronary artery abnormalities; CRP, C Reactive Protein; ALT, alanine aminotransferase, AST, Aspartate aminotransferase; FBC, Full blood count; Plt, Platelet count; Na, serum sodium; AMH, Archbishop Makarios Hospital; үGT, Gamma -Glutamyl Transferase; Hb, Hemoglobin; WBC, White Blood Cell count; ESR, Erythrocyte Sedimentation Rate; AHA, American Heart Association; AICc, Akaike Information Criterion; GI, Gastrointestinal; CNS, Central Nervous System; IVIG, Intravenous immunoglobulin; PPV, Positive predictive value; NPV, Negative predictive value;

\section{Declarations}


Ethics approval: Ethical approval was waived by the National Bioethics Committee as it was retrospective, completely anonymized and did not include any personal data.

Consent for publication: Not applicable

Availability of data and materials: Most important elaborated data generated and analysed during the study are included in this published article. Raw data will be available on request to the corresponding author.

Competing interests: The authors declare no known competing interests.

Funding: No funding was received for conducting this study

\section{Authors' contributions:}

Maria G. Koliou and Antonis Jossif designed the study and wrote the study protocol.

Maria G. Koliou, Elena Georgiou, Athina Aristidou, Marianna Kousparou, Avraam Elia and Maria Agathocleous collected data.

Maria G. Koliou, Athina Aristidou and Stella Mazeri analyzed the data and prepared the first draft of the manuscript.

Stella Mazeri advised for the statistical analysis and critically reviewed the data interpretation.

All authors have read and approved the final manuscript.

Acknowledgment: Not applicable

\section{References}

1. Jane W Newburger, Masato Takahashi, Michael A Gerber et al. Diagnosis, Treatment, and Long-Term Management of Kawasaki Disease: A Statement for Health Professionals from the Committee on Rheumatic Fever, Endocarditis, and Kawasaki Disease, Council on Cardiovascular Disease in the Young, American Heart Association.Circulation.2004;110:2747-71

10.1161/01.CIR.0000145143.19711.78

2. Kawasaki T. Infantile acute febrile mucocutaneous lymph node syndrome with specific desquamation of the fingers and toes. Clinical observation of 50 cases. Jpn J Allerg. 1967;178-222 (in Japanese with English abstract)

3. Dhrubajyoti Sharma, Surjit Singh. Kawasaki disease - A common childhood vasculitis. Indian J Rheumatol

4. . 2015 Dec;10: S78-S83.

5. Eileen Rife, Abraham Gedalia. Kawasaki Disease: an Update. Curr Rheumatol Rep. 2020 Sep 
6. Brian W McCrindle, Anne H Rowley, Jane W Newburger et al. Diagnosis, Treatment, and Long-Term Management of Kawasaki Disease: A Scientific Statement for Health Professionals from the American Heart Association. Circulation2017 Apr 25;135(17): e927-e999.

7. H Kato, S Koike, M Yamamoto, Y Ito, E Yano. Coronary aneurysms in infants and young children with acute febrile mucocutaneous lymph node syndrome. J Pediatr. 1975 Jun;86(6):892-8.

8. M Yanagisawa, N Kobayashi, S Matsuya. Myocardial infarction due to coronary thromboarteritis, following acute febrile mucocutaneous lymph node syndrome (MLNS) in an infant Pediatrics. 1974 Sep;54(3):277-80

9. Donato Rigante, Laura Andreozzi, Michele Fastiggi, Benedetta Bracci, Marco Francesco Natale, Susanna Esposito. Critical Overview of the Risk Scoring Systems to Predict Non-Responsiveness to Intravenous Immunoglobulin in Kawasaki Syndrome. Int J Mol Sci. 2016 Feb 24;17(3):278.

10. Elisa Fernandez-Cooke, Ana Barrios Tascón, Judith Sánchez-Manubens et al. Epidemiological and clinical features of Kawasaki disease in Spain over 5 years and risk factors for aneurysm development. (2011-2016): KAWA-RACE study group. PLoS One. 2019; 14(5): e0215665. Published online 2019 May 20. doi: 10.1371/journal.pone.0215665

11. Fan Yan, Bo Pan, Huichao Sun, Jie Tian, Mi Li. Risk Factors of Coronary Artery Abnormality in Children with Kawasaki Disease: A Systematic Review and Meta-Analysis. Front Pediatr. 2019 Sep 26; 7:374. doi: 10.3389/fped.2019.00374. eCollection 2019

12. R Core Team (2019). R: A language and environment for statistical computing. R Foundation for Statistical Computing, Vienna, Austria. URL https://www.R-project.org/)

13. H. Wickham. ggplot2: Elegant Graphics for Data Analysis. Springer-Verlag New York, 2016

14. Kamil Bartoń (2019). MuMIn: Multi-Model Inference. R package version 1.43.6. https://CRAN.Rproject.org/package=MuMIn

15. Georg Heinze and Meinhard Ploner (2018). logistf: Firth's Bias-Reduced Logistic Regression. R package version 1.23. https://CRAN.R-project.org/package=logistf to address issues of data separation

16. Mark Stevenson with contributions from Telmo Nunes, Cord Heuer, Jonathon Marshall, Javier Sanchez, Ron Thornton, Jeno Reiczigel, Jim Robison-Cox, Paola Sebastiani, Peter Solymos, Kazuki Yoshida, Geoff Jones, Sarah Pirikahu, Simon Firestone, Ryan Kyle, Johann Popp, Mathew Jay and Charles Reynard. (2019). epiR: Tools for the Analysis of Epidemiological Data. R package version 1.0-10. https://CRAN.R-project.org/package=epiR

17. A Alexopoulos, A Vekiou, L Lycopoulou, A Tavena, E Lagona, T Kakourou. Kawasaki disease in Greek children: a retrospective study. J Eur Acad Dermatol Venereol. 2013 May;27(5): 580-8.doi: 10.1111/j.1468-3083.2012.04488. x. Epub 2012 Feb 24

18. Uehara R, Belay ED.Epidemiology of Kawasaki Disease in Asia, Europe, and the United States. J Epidemiol. 2012;22(2):79-85. Epub 2012 Feb 4.

19. Yosikazu Nakamura, Mayumi Yashiro, Ritei Uehara et al. Epidemiologic Features of Kawasaki Disease in Japan: Results of the 2007-2008: Nationwide Survey. J Epidemiol. 2010;20(4):302-7. 
Epub 2010 Jun 5.

20. Li-Ping Xie, Wei-Li Yan, Min Huang et al. Epidemiologic Features of Kawasaki Disease in Shanghai from 2013 Through 2017.J Epidemiol. 2020; 30(10): 429-435.Published online 2020 Oct 5.

21. J Fukushige, N Takahashi, Y Ueda, K Ueda. Incidence and clinical features of incomplete Kawasaki disease. Acta Paediatr 1994 Oct;83(10):1057-60.

22. Jung Sook Yeom, Hyang Ok Woo, Ji Sook Park, Eun Sil Park, Ji-Hyun Seo, Hee-Shang Youn. Kawasaki disease in infants. Korean J Pediatr. 2013 Sep;56(9):377-82. Epub 2013 Sep 30.

23. Kimiyasu Egami, Hiromi Muta, Masahiro Ishii et al. Prediction of resistance to intravenous immunoglobulin treatment in patients with Kawasaki disease.J Pediatr. 2006 Aug;149(2):237-40.

24. Jane C Burns, Mary P Glodé. Kawasaki syndrome. Lancet. 2004 Aug 7-13;364(9433):533-44.

25. Adriana H Tremoulet, Brookie M Best, Sungchan Song et al. Resistance to intravenous immunoglobulin in children with Kawasaki disease. J Pediatr 2008 Jul;153(1):117-21. Epub 2008 Mar 4.

26. Tohru Kobayashi, Yoshinari Inoue, Kazuo Takeuchi et al. Prediction of intravenous immunoglobulin unresponsiveness in patients with Kawasaki disease. Circulation. 2006 Jun 6;113(22):2606-12. Epub 2006 May 30

27. Tetsuya Sano, Shunji Kurotobi, Kouji Matsuzaki et al. Prediction of non-responsiveness to standard high-dose gamma-globulin therapy in patients with acute Kawasaki disease before starting initial treatment. Eur J Pediatr. 2007 Feb;166(2):131-7. Epub 2006 Aug 1.

28. Lynn A Sleeper, L Luann Minich, Brian M McCrindle et al. Evaluation of Kawasaki disease risk-scoring systems for intravenous immunoglobulin resistance. J Pediatr. 2011 May;158(5):831-835.e3. Epub 2010 Dec 18.

29. Sarah Davies, Natalina Sutton, Sarah Blackstock et al. Predicting IVIG resistance in UK Kawasaki disease. Arch Dis Child. 2015 Apr;100(4):366-8. Epub 2015 Feb 10.

30. Andrea P Salgado, Negar Ashouri, Erika K Berry et al. High Risk of Coronary Artery Aneurysms in Infants Younger than 6 Months of Age with Kawasaki Disease. J Pediatr 2017 Jun; 185:112-116.e1. Epub 2017 Apr 10

31. Brian W McCrindle, Jennifer S Li, L LuAnn Minich et al. Coronary artery involvement in children with Kawasaki disease: risk factors from analysis of serial normalized measurements. Circulation. 2007 Jul 10;116(2):174-9. Epub 2007 Jun 18.

32. J W Newburger, M Takahashi, J C Burns et al. The treatment of Kawasaki syndrome with intravenous gamma globulin. N Engl J Med. 1986 Aug 7;315(6):341-7.

\section{Figures}




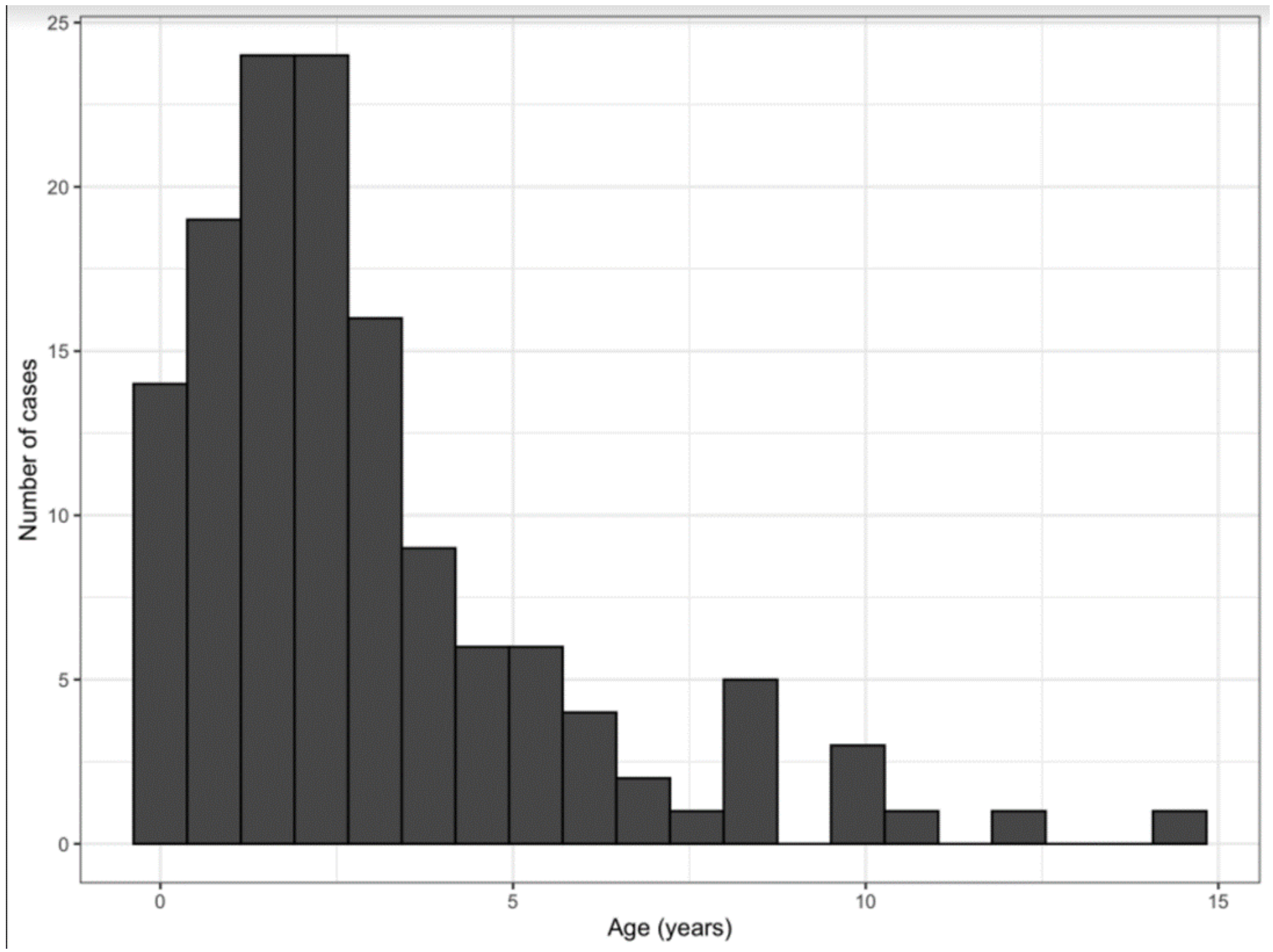

Figure 1

Age distribution of patients with KD 


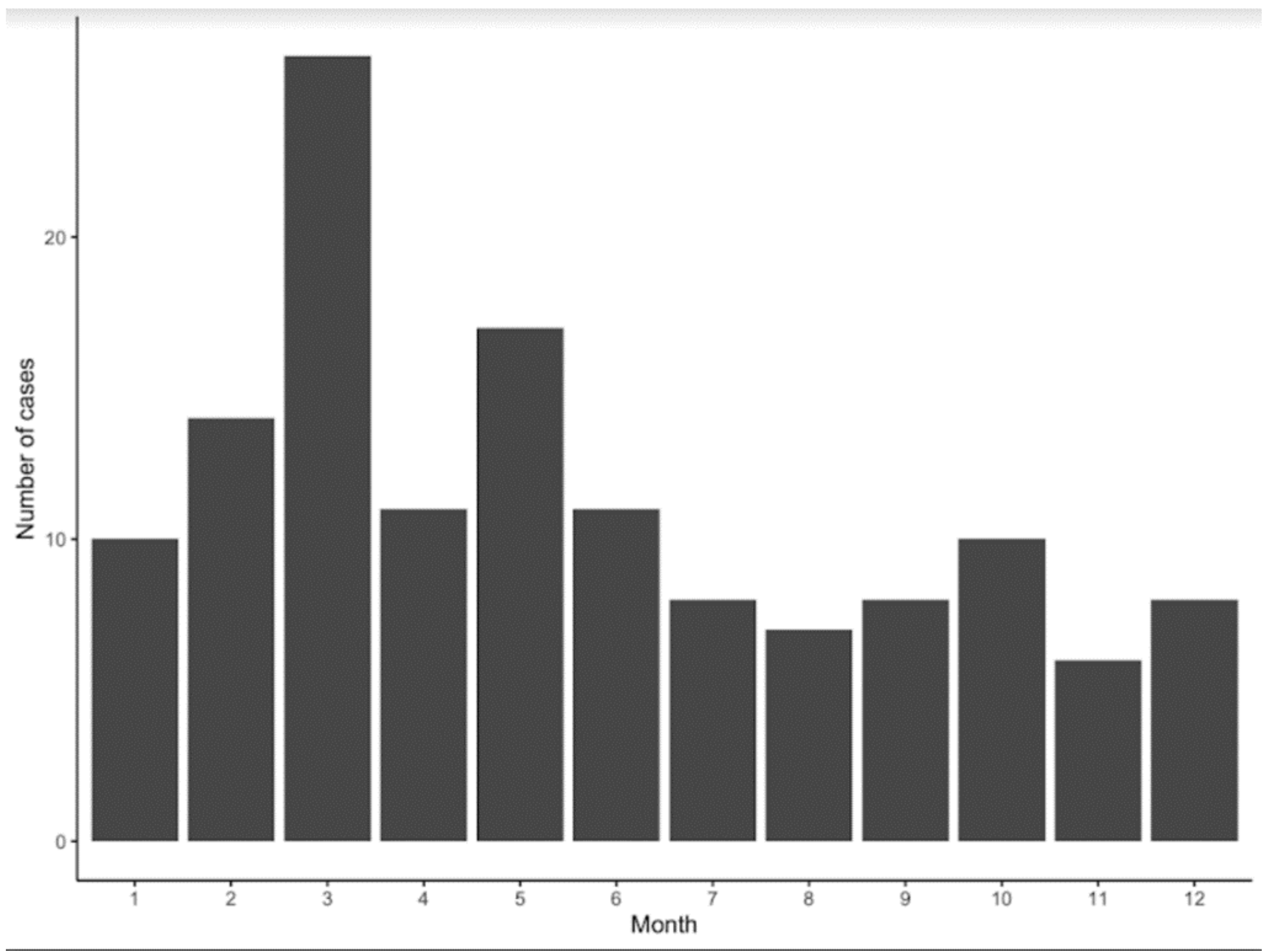

Figure 2

Distribution of patients with KD by time of admission 


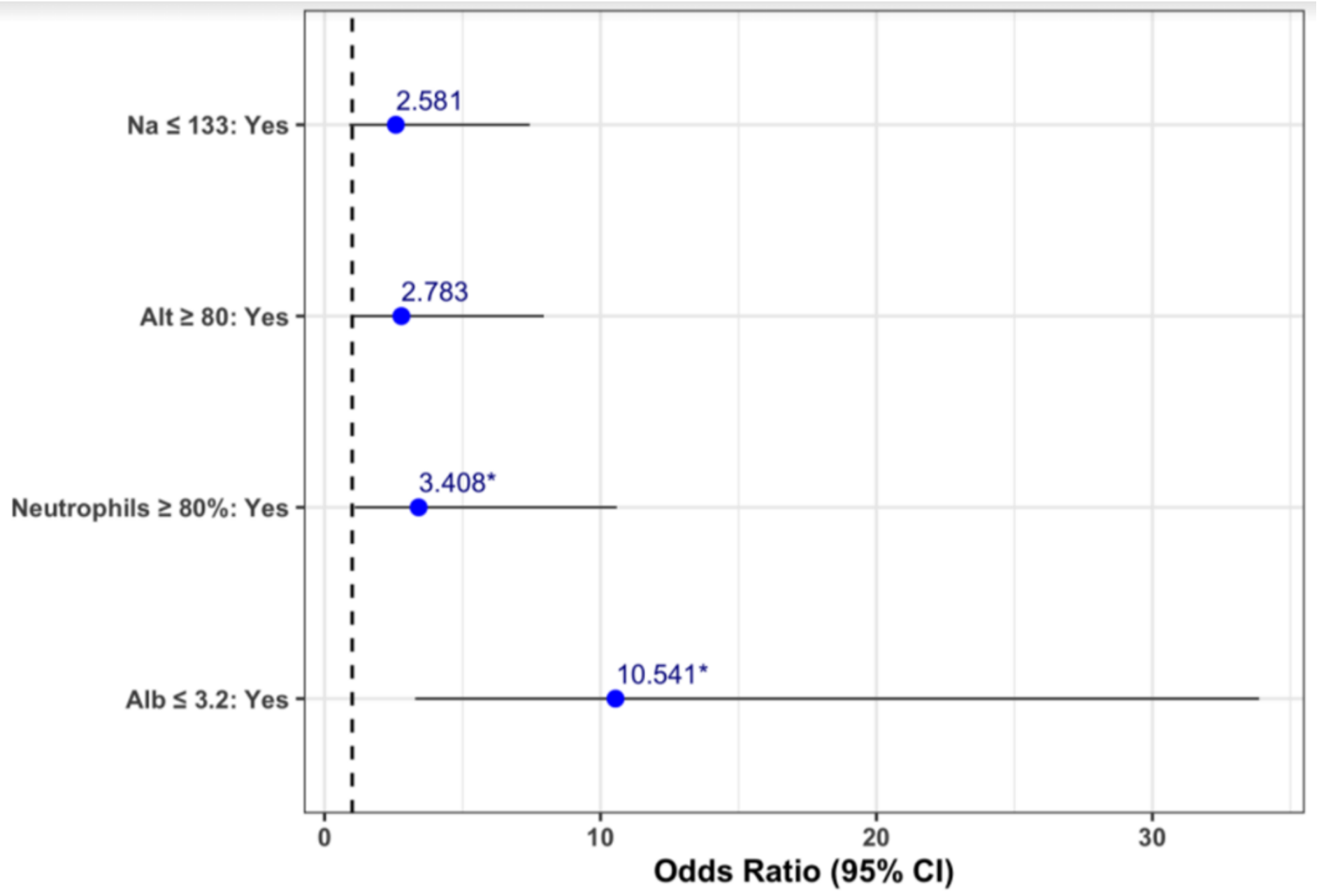

Figure 3

Multivariable logistic regression analysis of factors associated with IVIG non-response

\section{Supplementary Files}

This is a list of supplementary files associated with this preprint. Click to download.

- Additionalfile1.docx

- Additionalfile2.docx 